\title{
PERCEPÇÃO DE USUÁRIOS E NÃO USUÁRIOS DE FISIOTERAPIA EM RELAÇÃO À PROFISSÃO, EM LAURO DE FREITAS, BA
}

\author{
Charon de Magalhães Amado ${ }^{a}$ \\ Michele Castro Montoya Flores ${ }^{b}$ \\ Mansueto Gomes Neto ${ }^{\mathrm{C}}$
}

\begin{abstract}
Resumo
A origem da fisioterapia deu ênfase para as atividades de recuperação e reabilitação, decorrente das lesões ocorridas nas guerras mundiais. Apesar das mudanças de paradigmas ocorridos na profissão e o direcionamento da atuação profissional após a publicação da Classificação internacional de Funcionalidade a percepção da população sobre a atuação deste profissional ainda é influenciada pelo histórico da sua formação. Objetivo: avaliar a percepção de usuários e não usuários de fisioterapia sobre o que é, qual o seu papel e sua importância em Lauro de Freitas-BA. Método: Foi realizado um estudo descritivo com uma abordagem quantitativa, com amostra aleatória de pessoas residentes no município de Lauro de Freitas-BA Resultado: Foram entrevistadas 130 pessoas de ambos os sexos, maiores de 18 anos. O sexo feminino predominou em ambos os grupos com $68,46 \%$ do total. A maioria dos entrevistados afirmou saber o que é a fisioterapia, e que esse conhecimento se deu através de atendimento próprio ou através de um profissional de saúde. A maioria dos entrevistados afirmaram que a área de maior contato foi a de ortopedia. Conclusão: o conhecimento dos usuários e não usuários de em relação à fisioterapia ainda é restrito a reabilitação, com predominância especialmente na área ortopédica e que sua atuação se dá geralmente, quando a doença já se encontra instalada, acreditando-se fortemente nos recursos eletrotérmicos. sugerindo que o conhecimento esta de acordo com a experiência que o paciente teve durante o tratamento.
\end{abstract}

Palavras-chave: Fisioterapia; População; Conhecimento.

a. Bacharel em Fisioterapia pela União Metropolitana de Educação e Cultura (Unime), Lauro de Freitas, BA - Brasil. charonma.10@hotmail.com

b. Bacharel em Fisioterapia, especialista em saúde pública com ênfase em PSF, Docente Unime, Lauro de Freitas-BA, Brasil.micasflores@gmail.com

c. Bacharel em Fisioterapia, Mestre em Ciências da Reabilitação pela UFMG Doutor em Medicina e Saúde pela UFBA Professor adjunto do curso de Fisioterapia e do quadro permanente do Programa de pós Graduação em Medicina e Saúde da UFBA e docente da Unime Salvador, BA, Brasil. netofisio@gmail.com 


\title{
PERCEPTION OF USERS AND NON-USERS OF PHYSIOTHERAPY IN RELATION TO PROFESSION IN LAURO DE FREITAS, BA
}

\begin{abstract}
The origin of physiotherapy gave emphasis to the activities of recovery and rehabilitation as a result of the injuries occurred in world wars. Despite the changes in paradigms that have occurred in the profession and the direction of the professional practice after the publication of the international Classification of Functionality the perception of the population on the actuation of this professional is influenced by the history of their formation. Objective: Evaluate the perception of users and non-users of physiotherapy on what is, what its role and importance in Lauro de Freitas-BA. Method: a descriptive study was carried out with a quantitative approach, with random sample of persons residing in the city of Lauro of Freitas-BA. Result: We interviewed 130 people of both genders, aged over 18 years. Females predominated in both groups with $68,46 \%$ of the total. The majority of the interviewees experience what is the physical therapy and that this knowledge was through care or through a health professional. The majority of respondents reported that the area of greatest contact was the orthopaedics. Conclusion: the knowledge of the users and non-users in relation to the physical therapy is still restricted to rehabilitation, with predominance especially in orthopedic area and that their performance is generally, when the disease is already installed, it is believed strongly in electrical and thermotherapy resources suggesting that the knowledge of this agreement with the experience that the patient had during the treatment.
\end{abstract}

Keywords: Physical Therapy Specialty; Population; Knowledge.

\section{INTRODUÇÃO}

A percepção da população sobre a fisioterapia pode ser avaliada tanto pela utilização dos serviços, quanto a experiência por membros da família, amigos e até mesmo de uma comunidade. É importante para a profissão que a população entenda e conheça o que realmente é a fisioterapia e quais as suas especialidades..$^{(1-2)}$

Em 13 de outubro de 1969, foi então regulamentada a profissão do fisioterapeuta no Brasil. A situação atual da fisioterapia no Brasil é fruto da sua história, da sua evolução e conquista. Conhecer esse trajeto permite entender os problemas atuais que alertam sobre erros cometidos no passado. ${ }^{(3-6)}$
Num processo de construção do espaço profissional, o fisioterapeuta passou a atuar em diversas especialidades como: neurologia, geriatria, ortopedia, cardiologia, pediatria, ginecologia e obstetrícia, estética, ergonomia, pneumologia, oncologia entre outras ${ }^{(7-8-9)}$ Apesar de a fisioterapia ser uma profissão reconhecida no Brasil desde a década de 60 , há uma evidente falta de informação diante da população. ${ }^{(10)}$

Das atitudes e do comportamento profissional dos fisioterapeutas é que depende a profissionalização da fisioterapia, sendo que o grau de aceitação dessas atitudes pelo público também é de suma importância. O julgamento dos pacientes 
baseia-se em suas percepções, nos pontos em que se sentem mais qualificados para dar opinião. ${ }^{(9-10)}$

O conhecimento da população acerca do que é fisioterapia ainda é muito restrito ao atendimento clínico ou hospitalar. Enquanto o fisioterapeuta não identificar o seu papel e ser capaz de falar claramente sobre ele nas equipes multidisciplinares de saúde a profissão não irá avançar. ${ }^{(11)}$

A falta de estudos que descrevam o conhecimento da comunidade sobre a fisioterapia é um fator que contribui para parte do desconhecimento. ${ }^{(12)}$ Estudos contendo informações sobre a percepção e conhecimento da sociedade em relação a Fisioterapia podem contribuir para a adoção de medidas e estratégias de socialização do papel da fisioterapia para a população. Sendo assim, o objetivo geral do estudo foi avaliar e comparar a percepção de usuários e não usuários de fisioterapia sobre o que é, seu papel e sua importância em Lauro de Freitas-BA.

\section{MATERIAL E MÉTODOS}

Foi realizado um estudo descritivo e transversal, no município de Lauro de Freitas-Ba, no primeiro semestre de 2012. Uma amostra não probabilística foi composta por indivíduos de ambos os sexos, com idade maior que 18 anos, com pelo menos o ensino fundamental concluído, escolhidos de forma aleatória, convidados diretamente pelo entrevistador em diferentes bairros e locais da cidade. Foram excluídos estudantes e profissionais da área de saúde.

Os locais de coleta foram variados como: locais públicos (lojas, posto de gasolina, bancos, salões, academia, faculdade), vendedores ambulantes, residências.

Os objetivos e o procedimento do estudo foram explicados e aqueles que aceitaram participar da entrevista assinaram o TCLE elaborado baseado na resolução 196/96. Essa pesquisa foi aprovada e protocolada pelo comitê de ética em pesquisa da faculdade de tecnologia e ciências sob protocolo de registro $n^{\circ} 3614 / 2011$.
O participante respondiam a um questionário auto administrado, sendo possível tirar dúvidas quando preciso. Durante a coleta os indivíduos foram divididos em dois grupos de acordo com a realização ou não de atendimento fisioterapêutico prévio: os que nunca realizaram Fisioterapia $\left(G_{1}\right)$ definidos como não usuários e os que já tinham sido atendidos por fisioterapeutas $\left(G_{2}\right)$ definidos como usuários. Dois questionários contendo 23 questões objetivas de múltipla escolha foram aplicados, sendo um questionário para os que nunca realizaram Fisioterapia e o outro para os que já tinham sido atendidos por fisioterapeutas. Após as coleta os questionários foram separados de acordo com o grupo que o indivíduo se enquadrava.

As questões abordavam a identificação, renda familiar, nível de escolaridade, ocupação, conhecimento sobre a fisioterapia e sua importância na saúde da população, as áreas e recursos da fisioterapia mais conhecidos (utilizados), os locais de trabalho de um fisioterapeuta, o acesso ao serviço de fisioterapia, entre outras, algumas das questões foram destinadas as pessoas que já fizeram tratamento fisioterapêutico, e outras para aquelas que nunca fizeram fisioterapia, como: quais os motivos para nunca ter feito, se fosse fazer, qual área seria e em qual parte do corpo e o que é preciso para que a fisioterapia seja mais conhecida pela população.

Para analise dos dados demográficos e clínicos, foram utilizadas estatísticas descritivas. Os dados de variáveis contínuas foram analisados e apresentados como medidas de tendência central e dispersão e dados de variáveis dicotômicas ou categóricas foram analisados e apresentados como medidas de freqüência, com o uso de software SPSS (Statistical Package for the Social Science) for Windows (versão 14.0).

\section{RESULTADOS}

Foram entrevistadas 130 pessoas variando de 18 a 76 anos, destas $68,5 \%$ foram do sexo feminino. No $G 1$ (entrevistados que nunca realizaram fisioterapia - definidos como não usuários) foram entrevistadas 
62 pessoas com média de idade de $30,72 \pm 16,68$ anos, com $82,2 \%$ do sexo feminino, o nível de escolaridade predominante neste grupo foi o nível médio, já no grupo G2 (entrevistados que já foram atendidos por fisioterapeutas, definidos como usuários de
Fisioterapia) 68 pessoas foram entrevistadas, com média de idade de $48,51 \pm 12,61$ anos, sendo $55,9 \%$ do sexo feminino, a escolaridade predominante foi o nível médio. Os dados sócio-demográficos estão apresentados na Tabela 1.

Tabela 1. Características sócio-demográficas dos grupos

\begin{tabular}{ccc}
\hline & $\mathrm{G}_{1} \mathbf{N}=68$ & $\mathrm{G}_{2} \mathrm{~N}=6 \mathbf{2}$ \\
\hline SEXO & $\mathrm{n}(\%)$ & $\mathrm{n}(\%)$ \\
Feminino & $38(55,9)$ & $51(82,3)$ \\
\hline ESCOLARIDADE & & \\
Fundamental & $13(19,1)$ & $10(16,1)$ \\
Médio & $23(33,8)$ & $28(45,2)$ \\
Superior & $11(16,1)$ & $11(17,7)$ \\
RENDA FAMILIAR & & \\
Ol Salário & $17(25)$ & $12(19,4)$ \\
Ol a O3 Salários & $19(27,9)$ & $12(19,4)$ \\
Não respondeu & $17(25)$ & $29(46,8)$ \\
\hline
\end{tabular}

As respostas para as variáveis relacionadas ao conhecimento sobre fisioterapia; Sabe o que é a fisioterapia; Meio pelo qual passou a conhecer a fisioterapia; Como avalia o seu conhecimento sobre a fisioterapia; Quando ouve falar em fisiotera- pia associa a; Qual área da fisioterapia que procurou atendimento e se fosse procurar qual área seria para quem nunca fez fisioterapia estão expressas na Tabela 2.

Tabela 2. Conhecimento sobre a Fisioterapia

(continua)

\begin{tabular}{lcc}
\hline & $\mathrm{G} 1 \mathrm{~N}=\mathbf{6 8}$ & $\mathrm{G} 2 \mathrm{~N}=\mathbf{6 2}$ \\
\hline Sabe o que é? & $\mathrm{n}(\%)$ & $\mathrm{n}(\%)$ \\
$\operatorname{Sim}$ & $55(80,8)$ & $53(85,5)$ \\
Não & $\mathrm{O}$ & $\mathrm{O3}(4,8)$ \\
Mais ou menos & $13(19,1)$ & $\mathrm{O}(9,7)$ \\
\hline Como conheceu? & & \\
Através de profissional & $26(38,2)$ & $18(29,0)$ \\
Meios de comunicação & $\mathrm{O} 2(1,4)$ & $\mathrm{O3}(1,9)$ \\
Amigos & $\mathrm{O}(6,1 \%)$ & $27(43,5)$ \\
Atendimento & $31(45,6)$ & $\mathrm{O}$ \\
Outros & $\mathrm{O} 6(4,1)$ & $\mathrm{Ol}(\mathrm{O}, 6)$ \\
Ainda não conheço & $\mathrm{O}$ & $1 \mathrm{O}(16,1)$ \\
\hline
\end{tabular}




\begin{tabular}{|c|c|c|}
\hline & $\mathrm{G} 1 \mathrm{~N}=68$ & $\mathrm{G}_{2} \mathrm{~N}=62$ \\
\hline \multicolumn{3}{|c|}{ Como avalia seu conhecimento sobre? } \\
\hline Sabia o suficiente & $17(25 \%)$ & O \\
\hline Gostaria de saber mais & $49(72,1)$ & $46(74,2)$ \\
\hline Não acha necessário saber & O & $05(8,1)$ \\
\hline Outros & $\mathrm{O} 2(1,4)$ & $09(5,6)$ \\
\hline \multicolumn{3}{|l|}{$\begin{array}{l}\text { Quando ouve falar na Fisioterapia } \\
\text { associa a: }\end{array}$} \\
\hline Reabilitação & $48(70,6)$ & $27(43,5)$ \\
\hline Alongamento & $\mathrm{O} 8(11,8)$ & $13(20,9)$ \\
\hline Exercício & $07(10,3)$ & $13(20,9)$ \\
\hline Massagem & $\mathrm{O} 2(2,9)$ & O6(9,7) \\
\hline \multicolumn{3}{|l|}{ Procura por atendimento (área) } \\
\hline Ortopedia & $52(76,5)$ & $34(54,8)$ \\
\hline Neurologia & O8 $(11,8)$ & $\mathrm{O} 2(1,2)$ \\
\hline Dermato-funcional & O3 $(4,4)$ & $18(29,0)$ \\
\hline Uro-ginecologia & $\mathrm{O} 3(4,4)$ & $\mathrm{Ol}(\mathrm{O}, 6)$ \\
\hline Respiratória & $\mathrm{O} 2(1,4)$ & $07(11,3)$ \\
\hline Reumatologia & $\mathrm{Ol}(0,7)$ & O \\
\hline
\end{tabular}

Quando questionados sobre os recursos fisioterapêuticos, no $\mathrm{G}_{1}$ a maioria respondeu que os aparelhos eletrotérmicos com $52,94 \%$, seguidos de exercício com $35,29 \%$, massagens $8,82 \%$, pesos e bolas $2,94 \%$. No $\mathrm{G}_{2}$ 51,61\%, responderam aparelhos eletrotérmicos, seguidos de exercícios com
$30,64 \%$, massagem com $8,06 \%$, bolas e pesos com $4,83 \%$.

Na Figura 1 estão descritas as freqüências de respostas para o local de trabalho relatado pelos grupos.

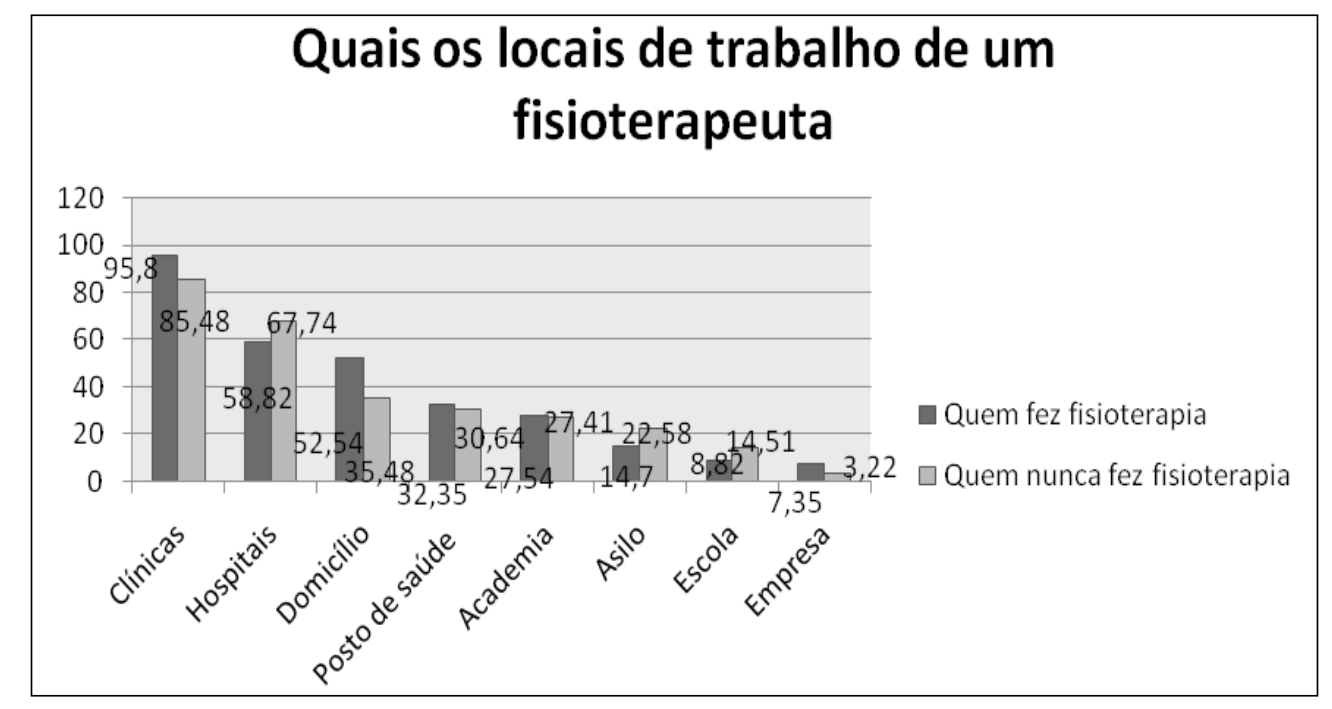

Figura 1. Locais de trabalho de um fisioterapeuta 
Sobre a importância da fisioterapia na saúde da população, no $\mathrm{G}_{1}$ 74,19\% mencionaram o item muito importante, $17,74 \%$ sem importância, $3,22 \%$ pouco importante, já no $\mathrm{G}_{2} 86,76 \%$ disseram ser muito importante e $11,76 \%$ disseram ser sem importância.

Quando questionados sobre qual público precisa de fisioterapia, no grupo de não usuários de Fisioterapia, idosos foram os mais indicados com $46,77 \%$, pacientes com lesão musculoesquelética com $43,54 \%$ e deficientes físicos com $41,93 \%$. Já no grupo dos usuários os mais votados foram deficientes físicos com $54,41 \%$, seguido de idosos com 47,05\% e pacientes com lesão musculoesquelética $42,64 \%$. Em ambos os questionários o item mencionado com menos de $2 \%$ foi pessoas sadias.

Quando perguntado ao $\mathrm{G}$ s sobre o motivo pelo qual nunca realizou um tratamento fisioterapêutico,
$74,19 \%$ porque nunca precisaram, $9,67 \%$ disseram por falta de vagas no SUS e porque o médico não recomendou; e outros motivos tiveram uma freqüência de $6,45 \%$. Dos que nunca precisaram $24,19 \%$ informaram que gostaria de já ter feito. A coluna com $35,48 \%$, seguido do corpo todo com $30,64 \%$, tornozelos e pernas com $14,51 \%$ e outros com $11,29 \%$, foram citados como a parte do corpo que gostaria de receber atendimento. Quando perguntados se atualmente precisam de tratamento fisioterapêutico 24,19\% disseram precisar.

Em relação ao item se gostaria que $o$ atendimento fosse realizado em casa, $67,74 \%$ disseram que sim, 22,58\% disseram que tanto faz e 9,67\% não.

$\mathrm{Na}$ Figura 2 estão apresentadas as freqüências em relação ao que é preciso para que a fisioterapia seja mais reconhecida pela população.

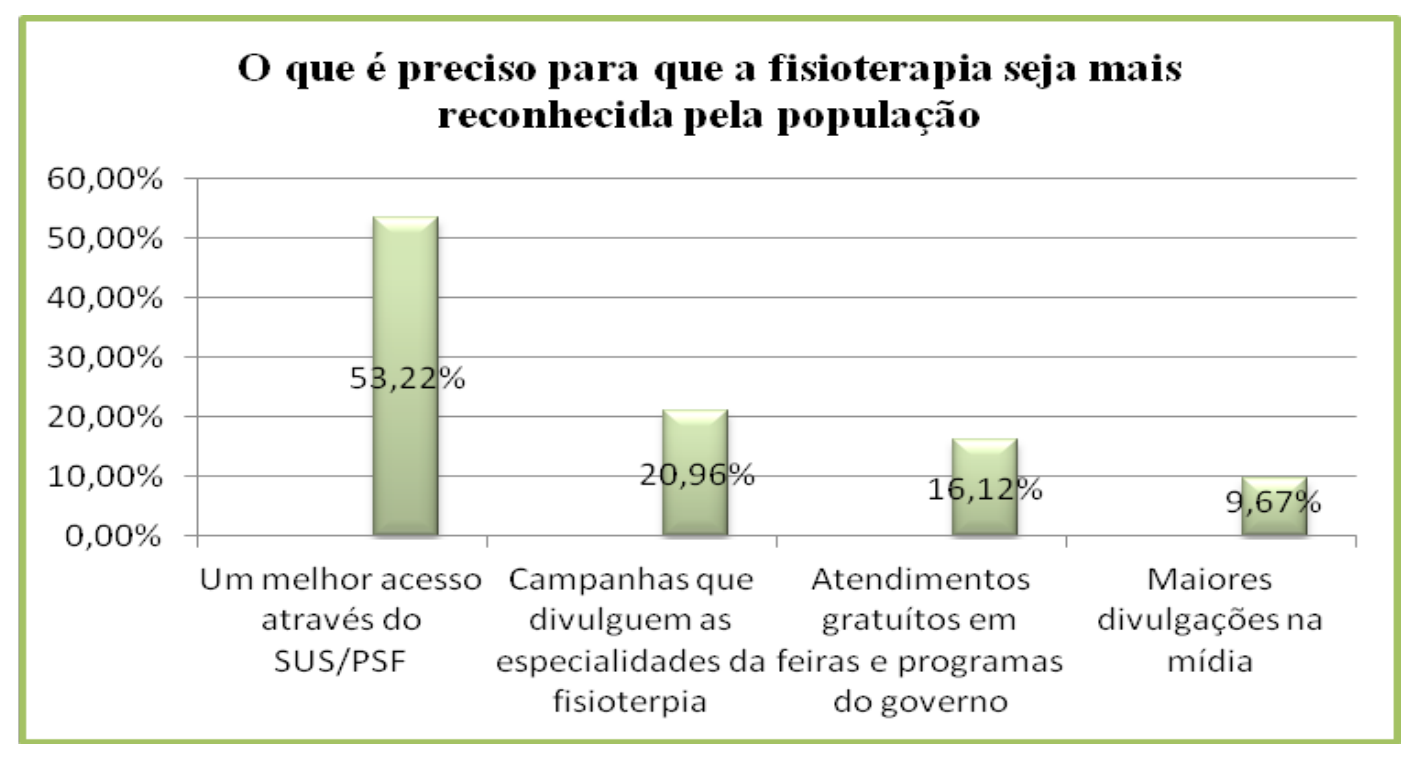

Figura 2. Reconhecimento da fisioterapia pela população

Para o grupo de usuários de Fiioterapia, foi perguntado sobre o grau de importância da fisioterapia na sua recuperação, $77,94 \%$ responderam muita, $16,17 \%$ pouca e $1,47 \%$ nenhuma e $100 \%$ dos entrevistados recomendariam a fisioterapia.
Na Figura 3 estão apresentadas as freqüências das respostas para a pergunta sobre os benefícios observados após a Fisioterapia. 


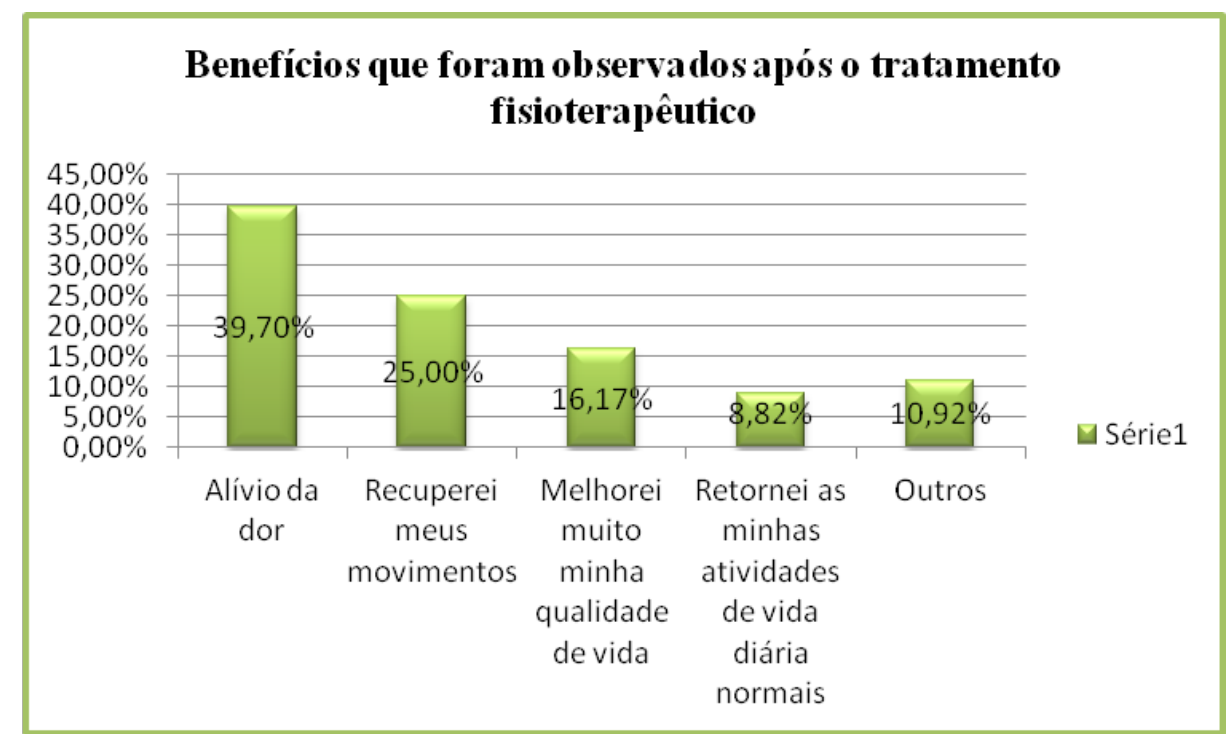

Figura 3. Benéficios da fisioterapia

Na relação com o Fisioterapeuta 95,58\% afirmaram que o fisioterapeuta é atencioso e 4,41\% responderam não, em relação ao esclarecimento de dúvidas no atendimento $80,88 \%$ responderam sim, $17,64 \%$ parcialmente e $1,47 \%$ disseram que não teve esclarecimento algum. Quando perguntados se faria novamente um tratamento fisioterapêutico, 92,64\% disseram que sim.

\section{DISCUSSÃO}

A maioria dos entrevistados foram do sexo feminino. No estudo de Nogueira e Machado os usuários do sexo feminino destacam-se em todos os serviços avaliados, ${ }^{(13)}$ assim como no estudo de Germano e Fayer em Juiz de Fora / MG. ${ }^{(14)}$

O fato de a maioria dos entrevistados já ter ouvido falar em fisioterapia é relevante, por tratar-se de uma profissão relativamente nova, embora não seja suficiente para garantir a qualidade dos conhecimentos construídos sobre a profissão. ${ }^{(15)}$

Para os usuários de fisioterapia o conhecimento em grande parte se deu através de atendimento próprio. Já para os não usuários o maior conhecimento se deu através de amigos e parentes, corroborando com o estudo de Carvalho (2008), em que $90 \%$ dos entrevistados afirmaram saber o que é a fisioterapia e que os profissionais de saúde foram os responsáveis por esse conhecimento. ${ }^{(15)}$ Apesar de afirmarem saber o que é a fisioterapia, a maioria respondeu que gostaria de saber mais, assim como no estudo de Carvalho (2008) sugerindo que essa divulgação ainda é superficial e, muitas vezes, de instância comercial. ${ }^{(15)}$

Quando perguntados sobre a que associam a palavra fisioterapia, a maioria respondeu reabilitação, confirmando que o fisioterapeuta é visto como um profissional reabilitador. As atribuições dos fisioterapeutas e dos profissionais de saúde, de um modo geral, estavam bastante restritas à doença. O modelo de saúde era o reabilitador. A prevenção de problemas e a promoção de saúde não faziam parte do leque de atuação do profissional. ${ }^{(16)}$

Gallo (2005) chama a atenção para o fato de que o fisioterapeuta, além de estar inserido no mesmo contexto dos demais profissionais da saúde com formação direcionada para a doença, padece desse infortúnio de forma mais acentuada, já que é visto como 'o profissional da reabilitação', ou seja, aquele que atua exclusivamente quando a doença, lesão ou disfunção já foi estabelecida. ${ }^{(17,18)}$

Os dois itens mais votados depois de reabilitação foram exercícios e alongamentos, mas o item massagem também foi mencionado. Acredita-se que, enquanto o fisioterapeuta não identificar $\circ$ 
seu papel e ser capaz de falar claramente sobre ele nas equipes multidisciplinares de saúde a profissão não irá avançar. ${ }^{(19)}$

Quando perguntado aos dois grupos sobre quais recursos fisioterapêuticos faziam parte do tratamento fisioterapêutico a maioria respondeu os aparelhos eletro-térmico, seguido exercício e massagem. Em um estudo similar de Carvalho (2008) a maioria dos entrevistados disse que o exercício e /ou a massagem ( $45 \%)$ e os aparelhos eletrotérmi$\cos (44 \%)$ são os principais recursos que o fisioterapeuta utiliza. ${ }^{(15)}$

No estudo de Machado e Fayer (2008), foi observado que $61,4 \%$ destes usuários foram tratados com equipamentos e apenas $8,8 \%$ com terapias manuais, porem a maioria destes equipamentos eletrotérmicos é coadjuvante no processo de tratamento. No entanto, sabe-se que tais equipamentos potencializam o aumento do volume de atendimentos, uma vez que o serviço consegue atender vários pacientes ao mesmo tempo. Pode-se justificar ainda a falta de acompanhamento durante as sessões, por parte do fisioterapeuta, que na intenção de ter um ganho maior (já que recebe financeiramente por paciente), procura por "meios terapêuticos" mais rentáveis. ${ }^{(14)}$

Sobre os locais de trabalho de um fisioterapeuta, para ambos os grupos, clínicas, hospitais e domicílio foram mais votados, seguidos de posto de saúde e academia. Na pesquisa de Carvalho (2008), o hospital foi o local mais votado com $59 \%$, seguido de clínica com 42\%. ${ }^{(15)}$ Para Ribeiro (2009) a fisioterapia caracterizou-se na formação de um profissional de função eminentemente reabilitadora, atuando primordialmente em centros de reabilitação e hospitais. ${ }^{(20)}$

A opção domicilio destina-se muito mais para aqueles que apresentam algum tipo de dificuldade de locomoção ou de deslocamento. $\mathrm{O}$ atendimento em casa seria algo cômodo e confortável para o paciente como afirma Carvalho (2008) em sua pesquisa, que a atividade domiciliar do fisioterapeuta foi citada por $36 \%$ dos entrevistados. ${ }^{(15-21)}$
A fisioterapia no domicilio é uma prática que vem crescendo muito em diversos países, como o Brasil. Dessa maneira, o profissional deixa a atuação tradicional em clínicas, consultórios e hospitais, para atingir clientes especiais que necessitam de atendimento em seu próprio domicílio. ${ }^{(22,23)}$ Neste novo paradigma, o fisioterapeuta passa a contribuir para uma melhoria nas ações de atenção à saúde nos diferentes níveis de complexidade. ${ }^{(24,25)}$

Quase 90\% dos usuários de Fisioterapia quando perguntadas sobre a importância da fisioterapia na sua recuperação, responderam que foi muito importante, onde apenas 10\% dizem ter tido pouca importância. Em relação ao principal benefício notado após o tratamento fisioterapêutico, o alivio da dor foi o mais citado, seguido de recuperação dos movimentos e melhora da QV, corroborando com o estudo de Ferreira (2009) que $56 \%$ dos pacientes em pesquisa referiram alivio da dor, seguido de melhora na postura. ${ }^{(26)}$ Para Marques et al (2002) A Fisioterapia tem um importante papel na melhora do controle da dor e no aumento ou manutenção das habilidades funcionais do paciente em casa ou no trabalho, assim como na redução de outros sintomas que lhe causam sofrimento. ${ }^{(27)}$

Mais de 100 entrevistados disseram que a fisioterapia é muito importante na saúde, demonstrando que a fisioterapia é valorizada pela população, assim como no estudo de carvalho que $90 \%$ dos entrevistados também consideraram muito importante. (15) Quando perguntado sobre o que era preciso para ser mais reconhecida pela população, grande parte dos entrevistados, responderam que o melhor acesso através do SUS/ESF/NASF, campanhas que divulguem suas especialidades, seriam as melhores opções.

A atuação do fisioterapeuta na ESF pode captar uma demanda reprimida da população que passa a usufruir do atendimento à medida que o fisioterapeuta é trazido para mais próximo das famílias. A Fisioterapia na ESF vai, ainda, prevenir o aumento do volume e complexidade da atenção em saúde, reduzindo os gastos públicos, colaborando com a mudança do modelo assistencial, ${ }^{(28)}$ impulsionando 
a mudança de perfil do profissional fisioterapeuta a não mais estar limitado a garantir sobrevida. ${ }^{(29,30)}$

Sugere-se que sejam feitos mais estudos com caráter qualitativo que possam identificar de forma clara e objetiva a opinião da população sobre o que é a fisioterapia e a percepção destes sobre a sua importância.

\section{CONCLUSÃO}

Pode-se perceber que o conhecimento da população em relação à fisioterapia ainda é restrito a reabilitação com predominância especialmente na área ortopédica e que sua atuação se dá geralmente, quando a doença já se encontra instalada, com a utilização de recursos eletrotérmicos. Acredita-se que a informação a cerca do que realmente é a fisioterapia e suas atribuições trariam benefícios à população no que diz respeito à promoção a saúde e a prevenção de doenças.

\section{REFERÊNCIAS}

1. Rebelatto JR, Botomé SP. Fisioterapia no Brasil. $2^{a}$ Ed. São Paulo: Manole; 1999.

2. IPEA - Instituto de Pesquisa Econômica Aplicada. SIPS- sistema de indicadores e percepção social. Rio de Janeiro; 2011.

3. Petri FC. História e interdisciplinaridade no processo de humanização da fisioterapia [dissertação]. Santa Maria, RS: Universidade Federal de Santa Maria; 2006.

4. Barros FBM. Poliomielite, filantropia e fisioterapia: o nascimento da profissão de fisioterapeuta no Rio de Janeiro dos anos 1950. Ciênc. saúde coletiva. 2008;13(3):941-954.

5. Rezende M, Moreira MR, Filho AA, Tavares MFL. A equipe multiprofissional da "saúde da família": uma reflexão sobre o papel do fisioterapeuta. Ciênc. saúde coletiva. 2009;14 supl.11403-1410.

6. Marques AP, Sanches EL. Origem e evolução da fisioterapia: aspectos históricos e legais. Rev. fisioter. Univ. São Paulo. 1994;1(1):5-10.
7. Brasil. Decreto Lei $n^{\circ} 938$, de 13 de outubro de 1969. Dispõe sobre as profissões de fisioterapeuta e terapeuta ocupacional.

8. Câmara AMCS; A formação e a atuação do profissional fisioterapeuta: um estudo com egressos da UFMG - 1982-2005 [dissertação]. Belo Horizonte: Universidade Federal de Minas Gerais; 2006.

9. Ann T. Fisioterapia de Tiddy. $12^{\mathrm{a}}$ Ed. São Paulo: Ed. Livraria Santos; 2002.

10. Deliberato PCP. Fisioterapia preventiva: fundamentos e aplicações. São Paulo: Manole; 2002.

11. Barros FBM. Autonomia profissional ao longo da história. Revista FisioBrasil. 2003;59:20-31.

12. Siqueira FV, Facchini AL, Hallal CP. Epidemiology of physiotherapy utilization among adults and elderly. Rev. saúde pública. 2005;39(4): 663-8.

13. Rodrigues RM, Raimundo CB, Silva KC. Satisfação dos usuários dos serviços privados de fisioterapia do município de campos dos Goytacazes-RJ. Perspectivas online. 2010 O4:(14)

14. Machado GLR, Fayer VA. Análise do perfil do serviço de fisioterapia do setor secundário do SUS de Juiz de Fora - MG [trabalho de conclusão de curso]. Juiz de Fora: Faculdade de Medicina da Universidade Federal de Juiz de Fora; 2008

15. Carvalho STRF. Conhecimentos e percepções dos usuários da estratégia saúde da família sobre fisioterapia [dissertação]. São Paulo: Faculdade de medicina de Ribeirão Preto;2008.

16. Faria L. As práticas do cuidar na oncologia: a experiência da fisioterapia em pacientes com câncer de mama. Hist. Ciênc. SaúdeManguinhos. 2010;17(supl.1):69-87.

17. Gallo DLL. A fisioterapia no Programa Saúde da Família: percepções em relação à atuação profissional e formação universitária. [dissertação]. Londrina: Universidade Estadual de Londrina; 2005.

18. Almeida, ALJ, Guimarães RB. O lugar social do fisioterapeuta brasileiro. Fisioter pesqui.2009;16(1):82-8. 
19. Nascimento MC, Sampaio R, Salmela JH, Mancini MC, Figueiredo IM. A profissionalização da fisioterapia em Minas Gerais. Rev. bras. fisioter.2006;10(2):241-247.

20. Ribeiro KSQS. A experiência na extensão popular e a formação acadêmica em fisioterapia. Cad. Cedes. 2009;29(79):335-346.

21. Costa JL, Pinho MA, Figueiras MC. A fisioterapia no programa de saúde da família: percepções dos usuários. Revista Ciência \& Saúde. 2009;2(1): 2-7.

22. Silva LWSS, Durães MA, Azoubel R. Fisioterapia domiciliar: pesquisa sobre o estado da arte a partir do Niefam. Fisioter mov. 2011;24(3):495501.

23. Delai KD, Winseiwsk MSW Inserção do fisioterapeuta no Programa Saúde da Família. Ciênc. saúde coletiva. 2011;16(Supl.1):1515-1523.

24. Torres CKD, Estrela JFM, Ribeiro KSQS. Contribuição da educação popular no atendimento fisioterapêutico domiciliar. Ciênc. saúde coletiva. 2009. 14(5):1877-1879.

25. Reichert N. O contexto de atuação profissional do fisioterapeuta na concepção dos usuários das unidades básicas de saúde do vale dos sinos [dissertação]. Novo Hamburgo: Centro Universitário Feevale; 2006.

26. Ferreira F. A avaliação da satisfação dos pacientes ortopédicos atendidos na Clínica Ortopédica da UNESC [monografia]. Criciúma: Universidade do Extremo Sul Catarinense, 2009.

27. Marques AP, Matsutani LA, Ferreira EAG; Mendonça LLF. A fisioterapia no tratamento de pacientes com fibromialgia: uma revisão de literatura. Rev.bras. reumatol. 2002;42(1):43.

28. Castro SS, Cipriano JG, Martinho A. Fisioterapia no Programa de Saúde da Família: uma revisão e discussões sobre a inclusão. Fisioter. mov. 2006;19(4):55-62.

29. Rodriguez MR. Análise histórica da trajetória profissional do fisioterapeuta até sua inserção nos Núcleos de Apóio a Saúde da Família (NASF) Comun. Ciênc. saúde. 2010;21(3):261266.

30. Aveiro MA, Acióli GG, Driusso P, Driusso P, Oishi J. Perspectivas da participação do fisioterapeuta no Programa Saúde da Família na atenção à saúde do idoso. Ciênc. saúde coletiva. 2011;16(Supl.1):1467-1478. 